\title{
Relationship between hysteretic behavior of magnetization and magnetoresistance in half-metallic ferromagnets
}

\author{
V. N. Krivoruchko, ${ }^{1}$ Y. Melikhov, ${ }^{2}$ and D. C. Jiles ${ }^{2}$ \\ ${ }^{1}$ Donetsk Physics \& Technology Institute, NASU, 72 R. Luxemburg Street, Donetsk, 83114, Ukraine \\ ${ }^{2}$ Wolfson Centre for Magnetics, Cardiff University, Newport Road, Cardiff CF24 3AA, Wales \\ (Received 21 April 2008; revised manuscript received 30 April 2008; published 20 May 2008)
}

\begin{abstract}
A generalized Preisach description of hysteretic magnetotransport properties of half-metallic ferromagnets (HMFs) is proposed. Assuming that the system consists of an assembly of elementary bistable hysterons distributed in energy levels and with a range of possible energy barriers, the connection between irreversible magnetic and transport properties of HMFs is found. Within this model, both the magnetization hysteresis and resistivity hysteresis of HMFs can be described by a few simple assumptions common to description of hysteresis phenomena leading to some fundamental relationship.
\end{abstract}

DOI: $10.1103 /$ PhysRevB.77.180406

PACS number(s): 75.60.Ej, 75.47.-m, 71.20.-b, 75.10.Lp

\section{INTRODUCTION}

Half-metallic ferromagnets (HMFs) are magnetic metals with an unusual band structure in which only states of one spin direction are present at the Fermi level, whereas there is a gap in the density of states for the other spin direction (for recent review, see Ref. 1). That is, in HMFs only the electrons (or holes) with spin, for example parallel of magnetization, are conducting while the charge carriers with the opposite spin direction are localized. There are several classes of potentially half-metallic materials, such as the hole-doped manganites $\mathrm{La}_{1-x} \mathrm{~A}_{x} \mathrm{MnO}_{3}$ (with divalent cation $A$ ), the double perovskites $\left(\mathrm{Sr}_{2} \mathrm{FeMoO}_{6}\right)$, the (semi)Heusler alloys (NiMnSb), magnetite $\left(\mathrm{Fe}_{3} \mathrm{O}_{4}\right)$, rutile $\left(\mathrm{CrO}_{2}\right)$, and others. Such materials are major components in devices of microelectronics, spin-electronics, and computing (for recent review, see Ref. 2) and they have received significant interest from researchers in the past decade. ${ }^{3-8}$ However, up to now a detailed understanding of the relation between irreversible magnetic and transport properties of these materials has been lacking. The proposed relations between magnetization and resistivity $^{7-11}$ are actually based on the lowest-order approaches and are valid only for a restricted range of parameters. The phenomenon has eluded a complete explanation and it is still a challenge to explain whether hysteresis in magnetization and hysteresis in magnetoresistivity of HMFs are mutually independent.

On thermodynamic grounds, hysteresis is a consequence of the fact that, when the system is not able to reach the thermodynamic equilibrium of a ground state during the time of the experiment, the system will remain in a temporary local minimum of its free energy, and its response to external actions will become history-dependent. ${ }^{12}$ Recently, particular attention has been focused on the description of combined hysteresis and thermal relaxation in magnetic materials. ${ }^{13,14}$ The working hypothesis is that the free energy of the system can be decomposed into the superposition of simple freeenergy profiles, each characterized by two energy minima separated by a barrier.

Generalizing these ideas, the connection between magnetoresistance hysteresis and magnetization hysteresis in HMF perovskite oxides is proposed in this Rapid Communication.
By assuming that the system consists of an assembly of elementary bistable units (magnetic hysterons) with a distribution of energy levels and energy barriers, a description of the hysteretic magnetoresistivity of HMFs is obtained. Direct relationships between magnetization hysteresis, $M(H, T)$, and magnetoresistivity hysteresis (or magnetoresistance), $R(H, T)$, of HMFs are obtained. Together with the predicted hysteretic response, the model is able to predict some specific relations in limiting cases between magnetization and magnetoresistance which have been experimentally observed for doped manganese perovskites.

\section{MODELING OF MAGNETORESISTIVE HYSTERESIS}

The widely used Preisach model ${ }^{12,15}$ assumes that the hysteretic system consists of a large number of elementary interacting units called hysterons. Each unit is described by an elementary rectangular hysteresis loop, which has two field parameters, i.e., switching fields $h_{A}$ and $h_{B}$, with $h_{A} \geq h_{B}$. A probability distribution function $P\left(h_{A}, h_{B}\right)$, which describes the probability density of the elementary units to have switching fields $h_{A}, h_{B}$, is assumed to be known. Under varying external magnetic field $H$, the magnetization $M$ of the unit will depend on the previous history of field changes. This is usually written using the operator $\gamma\left(H ; h_{A}, h_{B}\right)$, which may be \pm 1 depending on the relation between $H$ and $h_{A}, h_{B}$. The magnetization may be calculated knowing the history of variation of the magnetic field as

$$
M(H)=M_{S} \iint_{b(H)} \gamma\left(H ; h_{A}, h_{B}\right) P\left(h_{A}, h_{B}\right) d h_{A} d h_{B},
$$

where $M_{S}$ is the saturation magnetization, and the path $b(H)$ reflects the evolution of the magnetic field $H$ over time $t$ and divides the whole plane $h_{A} \geq h_{B}$ in two regions: in the first region, all elementary units are switched up and have the magnetization " +1 ," and in the second all units are switched down and have the magnetization " -1 ." We will assume that for the purposes of this calculation, the temperature is not too high, so that the thermal activation effects can be neglected.

We consider that the hysteretic system under study can be represented as an assembly of magnetic " +1 " and " -1 " hys- 
terons distributed in real space. The conductivity between two neighboring magnetic hysterons depends on many factors. In order to clarify how magnetic order can determine the transport properties of the system, the dependence of electron scattering on the relative orientation (parallel or antiparallel) of the magnetic moments of these neighboring hysterons only is considered. The description of the effect of magnetic order is based on the assumption that the electron scattering, which takes place at the interfaces between the magnetic clusters, depends only on the electron spin direction. This approach is very reasonable for HMFs, where it is known that electron scattering drastically depends on the electron spin direction.

So, we can consider a given magnetic hysteron distribution as a kind of transport resistor network. Following the concept behind the Preisach model, we consider a superposition of an infinite number of relay operators $R_{b(H)}$ $=R_{b(H)}\left(H ; h_{A i}, h_{B i} ; \ldots ; h_{A i+k}, h_{B i+k}\right)$-the simplest conductivity path. The switching fields $\left(h_{A i}, h_{B i}\right)$ are the corresponding fields for the $i$ th magnetic hysteron in the Preisach plane. Each $R_{b(H)}$ is weighted by a probability function $P\left(h_{A i}, h_{B i} ; \ldots ; h_{A i+k}, h_{B i+k}\right)$, which is a function introduced in this Rapid Communication, the Preisach conductivity function, which describes the probability of $k$ magnetic hysterons being involved in a conducting path. As a result, when the hysteresis is rate-independent, we obtain the following expression for an "irreversible" conductivity:

$$
\begin{aligned}
\sigma_{i r r}(H)= & \sigma_{s} \sum_{k=1}^{\infty} \iint_{b(H)} d h_{A i} d h_{B i} \\
& \times \iint_{b(H)} d h_{A i+k} d h_{B i+k} P\left(h_{A i}, h_{B i} ; \ldots ; h_{A i+k}, h_{B i+k}\right) \\
& \times R\left(H ; h_{A i}, h_{B i} ; \ldots ; h_{A i+k}, h_{B i+k}\right)
\end{aligned}
$$

where $\sigma_{s}$ is the saturation conductivity of a hysteron and $b(H)$ is the Preisach memory function, which is the same as for magnetic hysteresis. The correlation between magnetic and magnetoresistivity hysteresis can now be described by a correlation between the Preisach magnetic function $P\left(h_{A}, h_{B}\right)$ and the Preisach conductivity function $P\left(h_{A i}, h_{B i} ; \ldots ; h_{A i+k}, h_{B i+k}\right)$.

An important characteristic of the mixed-valence manganese perovskites exists that gives a physical background to suggest the fundamental relation between the conventional Preisach magnetic function, $P\left(h_{A}, h_{B}\right)$, and the newly proposed Preisach conductivity function, $P\left(h_{A i}, h_{B i} ; \ldots ; h_{A i+k}, h_{B i+k}\right)$, i.e., between the magnetization hysteresis and the magnetoresistivity hysteresis. Namely, within the double-exchange interaction model ${ }^{5-7}$ the itinerant charge carriers (electrons or holes) provide both the magnetic interaction between nearest $\mathrm{Mn}^{3+}-\mathrm{Mn}^{4+}$ ions and the material's electrical conductivity. Due to the short mean free path (that is typically the distance of about a lattice parameter), the charge carrier probes the magnetization on a very short length scale. As a result, for these systems a strong interplay between local magnetic order and macroscopic electrical resistance is expected.

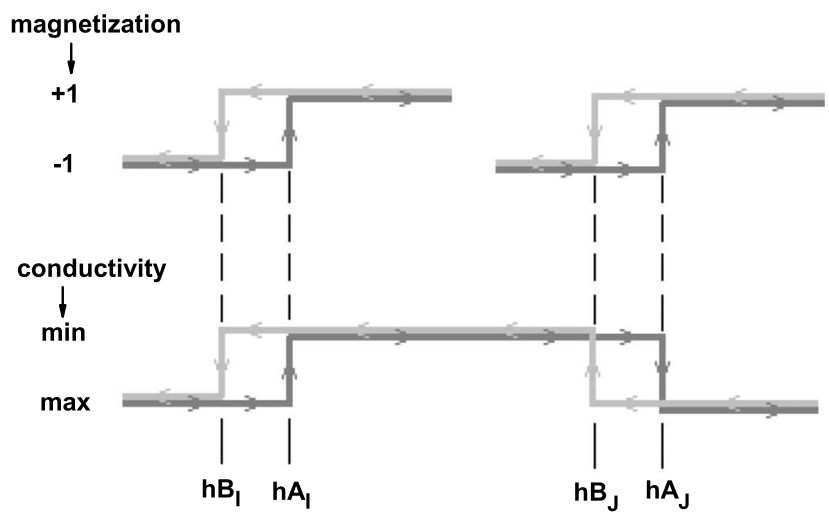

FIG. 1. Two magnetic hysterons $\left(h_{A i}, h_{B i}\right)$ and $\left(h_{A j}, h_{B j}\right)$ produce conductivity hysteron. Conductivity hysteron has two states with minimum and maximum conductivity with the switching fields coinciding with the magnetic hysteron.

Following the picture of a half-metallic magnetic material, we will suppose that the electron spin direction is parallel to the magnetic moment of the initial hysteron $\left(h_{A i}, h_{B i}\right)$ and may be parallel or antiparallel to the direction of the magnetic moment of the final hysteron $\left(h_{A j}, h_{B j}\right)$. If parallel, the electron experiences weak scattering and hence a low resistance $R_{\uparrow \uparrow}$ occurs (which we term the "conducting state"). If antiparallel, the electron experiences strong scattering and hence a high resistance, $R_{\uparrow \downarrow}$ occurs (which we term the "resistive state"). As an example, in Fig. 1, the resistive state arises in the field region $h_{A i} \leq H \leq h_{B j}$; hysteresis is possible for the regions of fields $h_{B i} \leq H \leq h_{A i}$ and $h_{B j} \leq H \leq h_{A j}$; for other magnetic field values, the conductivity hysteron is in the conductive state.

So, in general, one can give the following conceptual interpretation of the conductivity function $P\left(h_{A i}, h_{B i} ; \ldots ; h_{A i+k}, h_{B i+k}\right)$ : This distribution function describes the probability of $k$ magnetic hysterons being involved in a conducting path. This probability is proportional to both (i) the probability of the $\left(h_{A i}, h_{B i}\right) \cdots\left(h_{A i+k}, h_{B i+k}\right)$ magnetic hysteron being switched simultaneously and (ii) the probability of the hysterons in a virtual switching-fields plane to be nearest neighbors in real space. In the approximation of independent hysterons, the first probability is simply a product of the Preisach magnetic functions $P\left(h_{A i}, h_{B i}\right)$; the second probability is determined by the sample's construction. Depending on the structure of the Preisach conductivity function $P\left(h_{A i}, h_{B i} ; \ldots ; h_{A i+k}, h_{B i+k}\right)$, Eq. (2) is able to describe the resistive state in magnetic materials for any range of the system parameters. The wiping-out property ${ }^{12}$ (which is inherited in this model), the classical congruency property, ${ }^{12}$ as well as thermodynamics aspects of hysteresis for the system under consideration will be discussed in detail elsewhere.

In order to make qualitative predictions about the relations between the magnetization and magnetoresistivity, we consider the two limiting cases of the high-field and lowfield conductivity behavior.

(a) High-field limit $M \rightarrow M_{S}$. In the case of high magnetic alignment from grain to grain (low magnetic disorder), the isolated metallic regions are clustered and a global electrical 
connectedness can be achieved. All possible current trajectories through magnetic hysterons should be taken into account. That is, the function $P\left(h_{A i}, h_{B i} ; \ldots ; h_{A i+k}, h_{B i+k}\right)$ is then a multiple function of the probabilities $P\left(h_{A}, h_{B}\right)$ of any particular magnetic hysteron being involved in a conducting path,

$$
\begin{gathered}
P\left(h_{A i}, h_{B i} ; h_{A i+1}, h_{B i+1}\right)=c_{1} P\left(h_{A i}, h_{B i}\right) P\left(h_{A i+1}, h_{B i+1}\right), \\
P\left(h_{A i}, h_{B i} ; h_{A i+1}, h_{B i+1} ; h_{A i+2}, h_{B i+2}\right) \\
=c_{2} P\left(h_{A i}, h_{B i}\right) P\left(h_{A i+1}, h_{B i+1}\right) P\left(h_{A i+2}, h_{B i+2}\right), \\
P\left(h_{A i}, h_{B i} ; \ldots ; h_{A i+k}, h_{B i+k}\right)=c_{k} P\left(h_{A i}, h_{B i}\right) \ldots P\left(h_{A i+k}, h_{B i+k}\right),
\end{gathered}
$$

where $c_{1}, c_{2}, \ldots, c_{k}$ are constants, which cannot be ignored. For the given current trajectory, any mutual permutations of the hysterons are physically indistinguishable and have to be taken into account once, i.e., $c_{k}=$ const $/ k$ !, where "const" is determined by the distribution of the hysterons in real space. From Eq. (2), we then obtain $\sigma_{\text {irr }}(H) / \sigma_{s} \sim \exp \left[M(H) / M_{S}\right]$, and therefore, in terms of resistivity $R(H) / R(0) \sim \exp \left[-M(H) / M_{S}\right]$. A detailed study indicates that for good quality crystals, the resistivity and magnetization are related by the exponential dependence over a wide temperature range all the way up to the Curie temperature 7,16 (see Fig. 4 in Ref. 7 and Fig. 4 in Ref. 16).

(b) Low-field case $M \rightarrow 0$. In the limit of low magnetic alignment from grain to grain (strong magnetic disorder), the tunneling of carriers is mainly within a single pair of adjacent domains. The global electrical connectedness can be neglected and the magnetoresistance is associated with the mutual orientation of magnetization of neighboring grains. The likelihood of a conducting path involving a larger number of magnetic hysterons becomes negligibly small. The function $P\left(h_{A i}, h_{B i} ; \ldots ; h_{A i+k}, h_{B i+k}\right)$ can then be reduced to the probability of two magnetic hysterons $\left(h_{A i}, h_{B i}\right)$ and $\left(h_{A j}, h_{B j}\right)$ being involved in a conducting path,

$$
\begin{aligned}
P\left(h_{A i}, h_{B i} ; \ldots ; h_{A i+k}, h_{B i+k}\right) & \Rightarrow P\left(h_{A i}, h_{B i} ; h_{A j}, h_{B j}\right) \\
& =c_{1} P\left(h_{A i}, h_{B i}\right) P\left(h_{A j}, h_{B j}\right) .
\end{aligned}
$$

Simple calculations lead to the following relation between magnetization hysteresis $M(H)$ and conductivity hysteresis $\sigma(H)$ :

$$
\begin{aligned}
& \sigma_{\text {irr }}(H) / \sigma_{S} \\
& =\operatorname{const}\left(\iint_{b(H)} d h_{A i} d h_{B i} \gamma\left(H ; h_{A i}, h_{B i}\right) P\left(h_{A i}, h_{B i}\right)\right)^{2} \\
& \quad \sim\left[M(H) / M_{S}\right]^{2}
\end{aligned}
$$

or in terms of resistivity $R(H) / R(0)=1-\operatorname{const}\left[M(H) / M_{S}\right]^{2}$. Indeed, the data obtained on a series of manganite oxides demonstrate that in low magnetic field, the electric resistivity and the magnetization can be described by Eq. (5) (see Fig. 3 in Ref. 10).

Based on the relationships introduced above, we can construct both magnetic $M(H)$ and magnetoresistive $R(H)$ hysteresis loops if the proper probability function (Preisach
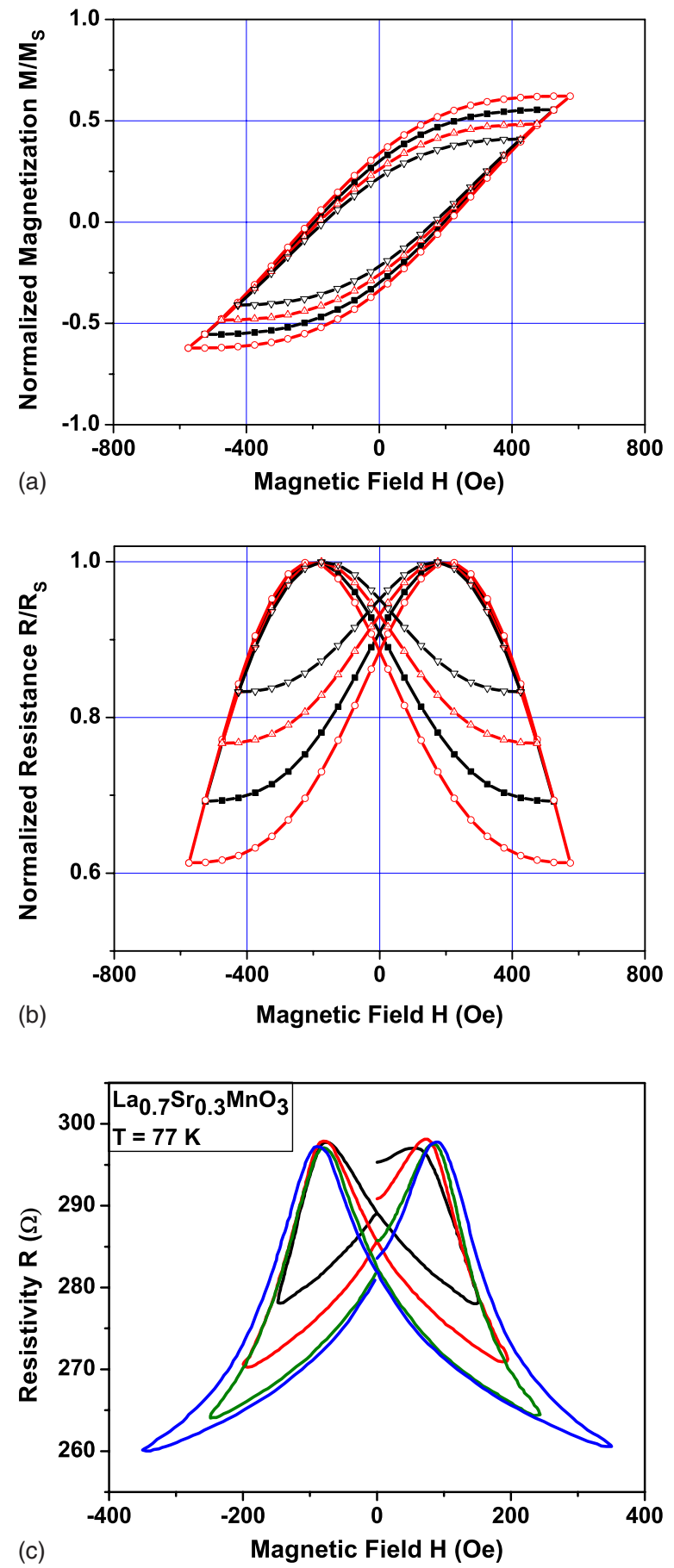

FIG. 2. (Color online) (a) Magnetization as a function of magnetic field curves constructed using classical Preisach model. (b) Resistivity as a function of magnetic field curves constructed using the proposed model. The following parameters for (a) and (b) were taken: $\sigma_{C}=300, \sigma_{I}=300, \bar{h}_{C}=100$, and the Preisach function was properly normalized. (c) Experimental resistivity as a function of magnetic field measured on $\mathrm{La}_{0.7} \mathrm{Sr}_{0.3} \mathrm{MnO}_{3}$ samples at $77 \mathrm{~K}$ (Ref. 17).

magnetic function) is known. As an example, Fig. 2(a) shows magnetic hysteresis loops calculated by Eq. (1) when the Preisach function is specified as a Gaussian-Gaussian distribution, ${ }^{12}$ 


$$
P\left(h_{C}, h_{I}\right)=\exp \left(-\frac{h_{I}^{2}}{2 \sigma_{I}^{2}}\right) \exp \left(-\frac{\left(h_{C}-\bar{h}_{C}\right)^{2}}{2 \sigma_{C}^{2}}\right),
$$

where $h_{C}=\left(h_{A}-h_{B}\right) / 2$ is a coercive field and $h_{1}=\left(h_{A}+h_{B}\right) / 2$ is an interaction field of the hysteron $\left(h_{A}, h_{B}\right)$, $\sigma_{C}$ and $\sigma_{I}$ are standard deviations of the coercive field and interaction field distributions, $\bar{h}_{C}$ is the average for the coercive field distribution, and the Preisach function is properly normalized. Figure 2(b) shows magnetoresistive hysteresis curves in a case in which the global electrical connectedness can be neglected and the magnetoresistance is associated with magnetization of neighboring grains. In this case, Eq. (5) can be used. The magnetoresistive hysteresis curves in Fig. 2(b) qualitatively reproduce the experimentally obtained curves on samples of compacted nanoparticles of $\mathrm{La}_{0.7} \mathrm{Sr}_{0.3} \mathrm{MnO}_{3}$ shown in Fig. 2(c) ${ }^{17}$

The low-field case $(M \rightarrow 0)$ opens (at least in principle) an interesting possibility to restore the distribution of the Preisach hysterons in real space. Indeed, from magnetic measurements one can easily restore the Preisach function $P\left(h_{A}, h_{B}\right)$. Then, fitting the model resistivity loops, such as in Fig. 2(b), to the experimental ones, such as in Fig. 2(c), one can restore the prefactor in Eq. (5). The last is directly the probability of the hysterons in a virtual switching-fields plane to be nearest neighbors in real space. This issue will be discussed in detail elsewhere.

In conclusion, a phenomenological approach to the description of both magnetization hysteresis and magnetoresistivity hysteresis is proposed on the basis of a few simple assumptions. Both types of hysteresis are interpreted assuming that the system consists of an assembly of elementary bistable units (hysterons), with a distribution of energy levels and energy barriers. The results obtained here provide a thermodynamic framework for the study of the connection between irreversible magnetic $M(H, T)$ and resistive $R(H, T)$ properties of HMFs. For systems like HMFs, we are able to predict the analytical dependence between different hysteretic properties of the system, such as Eqs. (3) and (4), based on the existence of unique distribution $P\left(h_{C}, h_{I}\right)$, which can be restored from magnetic measurements (see, e.g., Ref. 18).

\section{ACKNOWLEDGMENTS}

This research was supported by Cardiff University under the Visiting Fellow Scheme and by NAS of Ukraine under the Program "Nanostructured systems, Nanomaterials, Nanotechnologies."
${ }^{1}$ J. M. D. Coey, Materials for Spin Electronics, in Spin Electronics, edited by M. Ziese and M. J. Thornton (Springer, Berlin 2001).

${ }^{2}$ J. F. Gregg, I. Perej, E. Jouguelet, and C. Dennis, J. Phys. D 35, R121 (2002).

${ }^{3}$ S. Jin, T. H. Tiefel, M. McCormack, R. A. Fastnacht, R. Ramesh, and L. H. Chen, Science 264, 413 (1994).

${ }^{4}$ N. D. Mathur, G. Burnell, S. P. Isaac, T. J. Jackson, B.-S. Teo, J. L. MacManus-Driscoll, L. F. Cohen, J. E. Evetts, and M. G. Blamire, Nature 387, 266 (1997).

${ }^{5}$ J. M. D. Coey, M. Viret, and S. von Molnar, Adv. Phys. 48, 167 (1999).

${ }^{6}$ E. Dagotto, T. Hotta, and A. Moreo, Phys. Rep. 344, 1 (2001).

${ }^{7}$ M. B. Salamon and M. Jaime, Rev. Mod. Phys. 73, 583 (2001).

${ }^{8}$ M. Ziese, Rep. Prog. Phys. 65, 143 (2002).

${ }^{9}$ J. O’Donnell, M. Onellion, M. S. Rzchowski, J. N. Eckstein, and I. Bozovic, Phys. Rev. B 54, R6841 (1996).

${ }^{10}$ J. Fontcuberta, B. Martinez, A. Seffar, S. Piñol, J. L. GarciaMuñoz, and X. Obradors, Phys. Rev. Lett. 76, 1122 (1996).

${ }^{11}$ S. L. Yuan, Z. Y. Li, W. Y. Zhao, G. Li, Y. Jiang, X. Y. Zeng, Y.
P. Yang, G. Q. Zhang, F. Tu, C. Q. Tang, and S. Z. Jin, Phys. Rev. B 63, 172415 (2001).

${ }^{12} \mathrm{G}$. Bertotti, Hysteresis in Magnetism (Academic, New York, 1998).

${ }^{13}$ V. Basso, C. Beatrice, M. LoBue, P. Tiberto, and G. Bertotti, Phys. Rev. B 61, 1278 (2000).

${ }^{14}$ R. M. Roshko and C. A. Viddal, Phys. Rev. B 72, 184422 (2005).

${ }^{15}$ F. Preisach, Z. Phys. 94, 277 (1935).

${ }^{16}$ M. F. Hundley, M. Hawley, R. H. Heffner, Q. X. Jia, J. J. Neumeier, J. Tesmer, J. D. Thompson, and X. D. Wu, Appl. Phys. Lett. 67, 860 (1995).

${ }^{17}$ M. M. Savosta, V. N. Krivoruchko, I. A. Danielenko, V. Yu. Tarenkov, T. E. Konstantinova, A. V. Borodin, and V. N. Varyukhin, Phys. Rev. B 69, 024413 (2004); Yu. F. Revenko, M. M. Savosta, V. N. Krivoruchko, and I. A. Danielenko (unpublished).

${ }^{18}$ Ch. R. Pike, A. P. Roberts, and K. L. Verosub, J. Appl. Phys. 85, 6660 (1999). 